Cultura Física y Deporte

\title{
Evaluación del Vo2 máximo indirecto a través del test Course Navette en los oficiales de la Escuela Militar de Cadetes “General José María Córdova”
}

\author{
Luz Marina Umbarila Espinosa \\ Jhonathan Fernández Pinto \\ Leider Ortiz Ortiz
}

Escuela Militar de Cadetes “General José María Córdova”

\begin{abstract}
RESUMEN
El objetivo de la investigación es medir las capacidades físicas que tienen los oficiales de la Escuela Militar de Cadetes “General José María Córdova” (ESMIC en adelante), específicamente el Vo2máx, que se refiere a la capacidad que posee el ser humano de convertir el oxígeno en energía. Se trata de un estudio transversal, observacional y descriptivo de tipo cuantitativo, en el cual se empleó el test Course de Navette para realizar las mediciones. La muestra está compuesta por todos los oficiales de la ESMIC, quienes se dividieron en dos grupos: superiores y subalternos. Los resultados evidencian una diferencia significativa en el Vo2máx del valor esperado entre los grupos analizados (p < 0.001), con un $\mathrm{p}=1.68$, lo cual indica que el grupo de oficiales subalternos presenta significativamente una mayor resistencia en términos de Vo2máx.
\end{abstract}

\section{PALABRAS CLAVE}

Capacidades físicas; consumo de oxígeno; IMC; militares; salud.

\section{CITACIÓN}

Umbarilla Espinosa, L., Fernández Pinto, J. \& Ortiz Ortiz, L. (2020). Evaluación del Vo2 máximo indirecto a través del test Course Navette en los oficiales de la Escuela Militar de Cadetes “General José María Córdova”. Revista Brújula de Investigación, 8(16), 30-36. https://doi.org/10.21830/23460628.78 


\section{Introducción}

Las capacidades físicas son aquellas condiciones físicas con las que cuenta cada ser humano. Es decir que tanto las mujeres como los hombres cuentan con las mismas capacidades físicas, las cuales pueden perfeccionar por medio del entrenamiento (Chávez Pérez-Terán et al, 2015). Entre estas capacidades físicas se encuentran la potencia, la fuerza, la velocidad, la resistencia, el ritmo, el equilibrio y el acoplamiento, etc., con las cuales una persona podría realizar ejercicios o actividades sin problemas, llegado el caso una determinada acción motora (Sánchez, 2010). Por ejemplo, para que una persona pueda levantar una pesa de ciertos kilogramos y mantenerla por encima de la cabeza durante determinado tiempo debe contar con unas capacidades físicas, primordialmente la fuerza y la velocidad explosiva, que le permiten realizar el ejercicio (acciones motrices) en un tiempo mínimo; lo único que debe evitar es hacer un mal movimiento para no tener una lesión.

Ahora bien, las capacidades físicas se pueden medir utilizando distintos métodos y unidades de medida (Gutiérrez, 2010). La potencial consiste en todas las capacidades y habilidades con las que cuenta una persona o deportista y cuya medición, como las otras capacidades, se puede establecer en la ejecución de los diferentes ejercicios. Específicamente, en este trabajo se analiza la capacidad física de resistencia de los oficiales de la Escuela Militar de Cadetes “General José María Córdova” mediante el test de Course Navette.

Para los oficiales es fundamental tener una buena condición física en las capacidades básicas: fuerza, resistencia, flexibilidad y velocidad —reaccionar con la máxima rapidez frente a una señal—, las cuales trabajan entrelazadas para lograr un excelente rendimiento. En el ámbito militar es muy importante la resistencia, que depende de la capacidad máxima del organismo para metabolizar el oxígeno en la sangre. Esta capacidad aeróbica está en función del volumen máximo de oxígeno (Vo2máx), mediante el cual se determina la capacidad que tiene una persona de convertir oxígeno en energía. Este porcentaje varía según la edad y la condición física de la persona (Saborit, Valle, Montoliú, Martínez \& Nistal, 2010).

Se puede observar que la clasificación que se establece según las s etapas, donde se determina según la velocidad que logre una persona en un tiempo determinado. Como se mencionó, dado que estas capacidades varían según la edad y la condición física, no se espera que los oficiales de la ESMIC tengan el mismo resultado de Vo2máx ni que todos lleguen a una misma etapa. En consecuencia, el objetivo de este estudio es determinar mediante un test de Course Navette cuál es el nivel del Vo2máx indirecto en los oficiales y, con base en los resultados, buscar estrategias para que lo mejoren o mantengan.

El componente cardiorrespiratorio, que tiene una relación directa con la salud, ha sido ampliamente estudiado para determinar el rendimiento deportivo y la condición física del ser humano, independientemente de la edad o el sexo, y tiene una relación directa con el Vo2máx: "La máxima cantidad de oxígeno que el organismo es capaz de absorber, transportar y consumir por unidad de tiempo" (Lopez. Chicharo,2006) p. 409. Para hallar el Vo2máx existen dos formas: directa o indirecta. La medición del Vo2máx directa se realiza a través de un ergoespirómetro, pero la máquina utilizada para realizar esta prueba es muy costosa y difícil de adquirir. La forma indirecta, mucho más fácil, consiste en delimitar un área que mida 20 m, una ayuda sonora y una tabla de baremación para determinar los recorridos que realiza 
la persona evaluada. De acuerdo con el protocolo actual, la velocidad inicial es de 8,5 kmh-1 y se incrementa $0,5 \mathrm{kmh}-1$ cada minuto. En este estudio se empleó este método indirecto.

Es muy importante saber en qué condiciones físicas se encuentran los oficiales en la Escuela Militar, ya que su rendimiento físico es decisivo cuando son evaluados en pruebas físicas o cuando realizan actividades militares, como el entrenamiento militar con la tropa. Además, para los militares es determinante tener un buen rendimiento en la capacidad física de resistencia, debido a que sus actividades están basadas en la capacidad que tenga de resistir la exigencia como soldado de la patria. Desde la perspectiva de la educación física, la resistencia es la capacidad de realizar un esfuerzo de mayor o menor intensidad durante el mayor tiempo posible (Morehouse \& Miller, 1986), ya que la resistencia es la capacidad que posee una persona para soportar la fatiga en cualquier actividad. Según el tipo de actividad que se efectúe, se puede hablar de resistencia a la velocidad, resistencia a la fuerza, etc. (Mirella, 2002).

\section{Marco teórico}

Antes de empezar un entrenamiento es necesario saber la condición física en que se encuentra el individuo. Específicamente, la resistencia se puede hallar por medio del test de Course Navette, para obtener la potencia aeróbica máxima y relacionar dichos resultados con la actividad física y la salud. Este test consiste en medir la potencia aeróbica máxima (PAM) y el consumo máximo de oxígeno (Vo2máx.) expresado en litros por minuto $(\mathrm{l} / \mathrm{m})$ o en mililitros por kilogramo por minuto (ml./ kg./ min.): cuanto mayor sea el resultado, mayor capacidad tendrá el organismo para producir energía. Para determinar este resultado se emplea la tabla de baremación, la cual está compuesta por 20 etapas, cada una de las cuales está caracterizada por la velocidad que puede alcanzar y la distancia que puede recorrer la persona. Al aplicarse el test se obtiene la distancia recorrida y la velocidad alcanzada, las cuales se emplean en una fórmula para conocer el Vo2máx.

En 1982, Leger y Lambert crearon un test para determinar de manera indirecta el gasto máximo de oxígeno. Los sujetos debían recorrer un espacio de 20 metros consiguiendo una cadencia que aumentaba progresivamente. La velocidad inicial era de ocho kilómetros por hora, y extendía medio kilómetro por hora cada dos minutos hasta que los sujetos no lograban seguir el ritmo y se paraban (Leger \& Lambert, 1982). Esta velocidad máxima aeróbica y la edad del sujeto se introducían en una fórmula que proporcionaba de manera indirecta el consumo máximo de oxígeno.

Posteriormente, en 1988 Leger publicó la fórmula que se emplea actualmente para determinar el Vo2máx. a partir de la Course Navette. La fórmula es

$$
\begin{gathered}
\text { Vo2máx. }=31.025+3.238 X-3.248 A+ \\
0.1536 A X, \\
\text { siendo } X \text { la velocidad a la que se paró el } \\
\text { sujeto y A la edad. }
\end{gathered}
$$

Para sujetos mayores de 18 años, siempre se aplica el valor 18, de modo que la fórmula es

$$
\text { Vo2máx. =-27,4 + 6,0X, }
$$

siendo $X$ la velocidad a la que se paró el sujeto.

Con estas fórmulas y los valores que se obtuvieron en el test de Course de Navette se determinó el Vo2máx de los oficiales de la ESMIC, el cual se les di o a conocer con el fin de que tuvieran una perspectiva de mejora.

Ahora bien, es importante señalar que diversos estudios han comprobado que el organismo 
se adapta de diferentes formas al ejercicio en ambientes termo neutro "es decir, en ambientes con temperaturas suaves y en ambientes fríos, de manera que los resultados de estas pruebas varían En primer lugar, respecto a la termorregulación) señala que cuando una persona hace ejercicio en un ambiente frío tiene mayor pérdida de calor, tanto radiante como a través de la respiración, lo cual implica una menor temperatura interna. Así mismo, su temperatura muscular y el umbral de la temperatura para comenzar a sudar son menores, dado que desprende mayor calor como adaptación para regular la temperatura interna”. (Doubt, 1991).

En segundo lugar, en cuanto a las adaptaciones inmediatas cardiopulmonares, reporta que

Por último, de acuerdo en la dinámica metabólica para la producción de energía en este contexto térmico frío se observa que el organismo tiene una mayor tendencia a usar los sustratos glucólicos — tanto de la sangre como de los almacenes musculares-glucógeno- - y que hay un descenso relativo del empleo de ácidos grasos libres. Este hecho deriva en una mayor producción de lactato en iguales intensidades que el ejercicio, dado que el consumo de oxígeno es menor y, por ende, se deberá usar en mayor cantidad este principio inmediato en condiciones anaeróbicas para la producción de energía.

\section{Métodos}

Se trata de un estudio transversal, observacional, descriptivo y de tipo cuantitativo. Debido a su naturaleza, se siguió la Declaración de Helsinki y la Resolución 08430 de 1993 del Ministerio de Salud de Colombia. El macroproyecto del cual forma parte cuenta con aprobación del comité de ética interno con acta número 4363, y cada participante firmó el debido consentimiento informado. El universo y la muestra de la investigación está conformado por los
81 oficiales que componen la planta de personal de la ESMIC. La toma de datos se realizó de acuerdo con el protocolo del test Course Navette (Ruiz, 2006).

\section{Protocolo}

Para calcular el Vo2máx de los oficiales superiores y subalternos de la ESMIC se aplicó como método indirecto la prueba de resistencia Course de Navette, con la cual se mide la potencia aérobica máxima funcional mediante señales sonoras y una superficie plana con una distancia de 20 metros y demarcada de un lado a otro. Como se señaló, la prueba Course Navette o carrera de 20 metros es un test de aptitud cardiorrespiratoria que mide la potencia aeróbica máxima e indirectamente el consumo máximo de oxígeno, de modo que se trata de un test máximo y progresivo.

En el test, los oficiales de la ESMIC comienzan corriendo de un punto a otro situado a veinte metros de distancia al ritmo indicado por una señal sonora, que se va acelerando progresivamente cada minuto. En el momento en que suena la señal, deben haber llegado al otro punto para tocar la línea con el pie y encaminarse al punto inicial, al cual también deben llegar cuando vuelva a sonar la señal, y así sucesivamente. Durante la prueba, el oficial es el encargado de determinar su propio ritmo, de modo que se encuentre en un extremo de la pista al oír la señal, con una aproximación de 1 o 2 metros. La resistencia cardiorrespiratoria se deduce del momento en que el oficial interrumpe la prueba (Torres-Luque, 2014).

Los sujetos empiezan el test a una velocidad de ocho kilómetros por hora, el primer minuto aumenta a nueve kilómetros por hora y, a partir de ahí, cada minuto aumenta el ritmo medio kilómetro por hora hasta alcanzar 18 kilómetros por hora. Sin embargo, como se señaló, 
la prueba finaliza cuando no pueden seguir el ritmo marcado.

\section{Resultados}

La información sobre la muestra se presenta a continuación en la figura 1 .

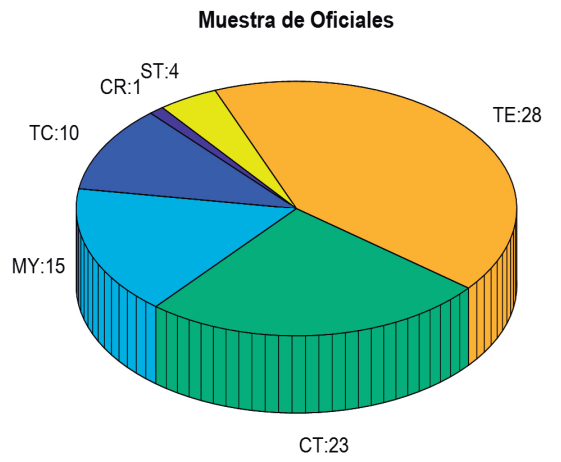

Figura 1. Muestra de oficiales por grado militar. CR.: Coronel. TC.: Teniente Coronel. MY.: Mayor. CT.:Capitán. TE.:Teniente. ST.:Subteniente.

Fuente: Elaborado por los autores.

Para el análisis se definieron dos grupos y se retiraron de la muestra a las personas con registros incompletos debido a criterios de inclusión y exclusión. Por lo tanto, se tiene:

- Grupo 1: formado por los oficiales superiores, que comprende los grados My., TC. y CR. (n1 = 26).

- Grupo 2: formado por los oficiales subalternos que comprende los grados ST., TE., CT. $(\mathrm{n} 2=55)^{1}$.

A continuación, la figura 2 muestra la distribución según sexo de la muestra seleccionada.

1 Se encontró evidencia estadística significativa de que el valor esperado de la flexibilidad es diferente entre grupos, siendo mayor en el grupo de oficiales subalternos $(p=0.0161)$.
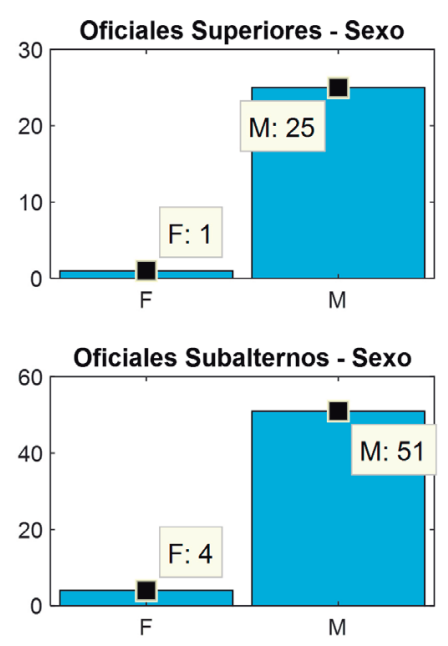

Figura 2. Histograma por sexo en el grupo 1 (oficiales superiores) y grupo 2 (oficiales subalternos). Fuente: Elaborado por los autores.

El análisis de significancia del Vo2máx (figura 3) evidencia que la media para oficiales superiores fue 41,95 y la de oficiales subalternos, 45,96. Como se observa, la desviación estándar para oficiales superiores es 3,73, mientras que para los oficiales subalternos es de 4,50. En términos de Vo2máx, los resultados muestran una diferencia estadística significativa del valor esperado entre los grupos analizados ( $\mathrm{p}<$ 0,001 ), con un $\mathrm{p}=1.68$. Esto representa que el grupo de oficiales subalternos presenta significativamente una mayor resistencia en términos de Vo2máx.

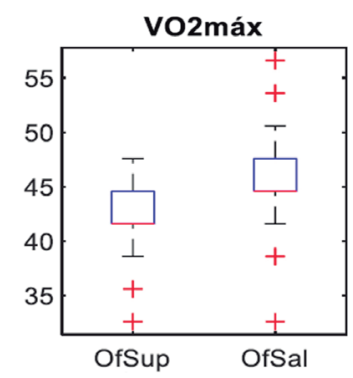

Figura 3. Análisis de significancia del Vo2máx. Fuente: Elaborado por los autores. 


\section{Discusión}

El objetivo de este estudio fue identificar el nivel de Vo2máx en los oficiales superiores y subalternos de la ESMIC. Los resultados parecen señalar que los oficiales superiores tienen tendencia a ser sedentarios, lo cual explicaría que su Vo2máx tienda a ser deficiente. En contraste, los oficiales subalternos realizan actividad física de forma más activa, lo cual les permite una mejor y mayor entrega de oxígeno a los diversos tejidos del cuerpo humano, sobre todo al tejido muscular, al realizar cualquier actividad física (Inbar, 1994).

Asimismo, el análisis estadístico confirma el resultado esperado de que los oficiales subalternos tuvieran mejor Vo2máx, ya que debido a la constante actividad física que implica su entrenamiento desarrollan la capacidad de aprovechar la mayor cantidad de oxígeno cuando respiran mientras corren o hacen otras actividades.

Desde otro punto de vista, los resultados indican que la capacidad del Vo2máx de los oficiales superiores es deficiente. Al comparar su edad (entre 35 y 45 años) y el resultado de Vo2máx que obtuvieron se encuentra que tienen un nivel regular, cuando se esperaría que tuvieran un nivel bueno o rozando el muy bueno. Por su parte, el rango de edad de los oficiales subalternos está entre los 22 y los 35 años, de modo que sus resultados de Vo2máx los ubican en un nivel bueno, con una tendencia a muy bueno. Como se ve, los oficiales de rango superior deben mejor su Vo2máx.

\section{Conclusión}

$\mathrm{Al}$ analizar los resultados genera gran impacto el nivel en el que se encuentran los oficiales superiores y subalternos. El Vo2máx muestra diferencia estadística significativa del valor esperado entre los grupos analizados $(\mathrm{p}<$
0.001), con un $\mathrm{p}=1.68$, lo cual evidencia que el grupo de oficiales subalternos presenta significativamente una mayor resistencia en términos de Vo2máx.

\section{Agradecimientos}

Los autores agradecen a la Escuela Militar de Cadetes “General José María Córdova”.

\section{Declaración de divulgación}

Los autores declaran que no existe ningún potencial conflicto de interés relacionado con el texto. Los puntos de vista y los resultados de este artículo pertenecen a los autores y no reflejan necesariamente los de la Escuela Militar de Cadetes “General José María Córdova”.

\section{Financiamiento}

Los autores no declaran fuente de financiamiento para la realización de este artículo.

\section{Sobre los autores}

Jhonathan Fernández Pinto es Oficial del Ejército Nacional de Colombia.

Leider Ortiz Ortiz es Oficial del Ejército Nacional de Colombia.

Luz Marina Umbarila Espinosa es magíster en Ciencias y Tecnologías del Deporte y la Actividad Física, Universidad Manuela Beltrán docente e investigadora del grupo RENFIMIL, Facultad de Educación Física Militar, Escuela Militar de Cadetes "General José María Córdova”, Bogotá, D. C., Colombia. Contacto: luz. umbarila@esmic.edu.co.

\section{Referencias}

Chávez Pérez-Terán, M., López Rosabal, F., Castro Gutiérrez, Y., Garrote Santana, H., Agramonte Llanes, O. M., Simón Pita, A. M., ... \& Lam Díaz, R. M. (2015). Biometría hemática en el control médico del entrenamiento de deportistas cubanos 
de alto rendimiento. Revista Cubana de Hematología, Inmunología y Hemoterapia, 31(1), 41-52.

Doubt, T. J. (1991). Physiology of exercise in the cold. Sports Medicine, 11(6), 367-381.

Gutiérrez, F. G. (2010). Concepto y clasificación de las capacidades físicas. Revista de Investigación Cuerpo, Cultura y Movimiento, 1(1), 77-86.

Inbar O, O. A. (1994). Normal cardiopulmonary responses during incremental exercise in 20- to 70-year-old men. Medi \& Sci en Deporte y Ejercicio.

Leger, L. \& Lambert, J. (1982). Maximal multistage 20-m shuttle run test to predict Vo2max. Eur J Appl Physiol, 49(1). 1-12.

Leger, L. A., Mercier, D., Gadoury, C., \& Lambert, J. (1988). The multistage 20 metre shuttle run test for aerobic fitness. Journal of sports sciences, 6(2), 93-101.

López Chicharro, J., \& Fernández Vaquero, A. (2006). Fisiología del ejercicio.
Mirella, R. (2002). Las nuevas metodologías del entrenamiento de la fuerza, la resistencia, la velocidad y la flexibilidad. Barcelona: Paidotribo.

Morehouse, L. \& Miller, A. T. (1986). Fisiología del ejercicio. Buenos Aires: El Ateneo.

Ruiz, M. P. (2006). Pruebas funcionales de valoración aeróbica. En López Chicharro J, Vaquero F. (Eds.), Fisiología del ejercicio (3ª. ed.). Buenos Aires: Editorial Panamericana.

Saborit, J., Valle, M., Montoliú, M., Martínez, P. \& Nistal, P. Y. (2010). Relación entre la percepción de la capacidad aeróbica y el Vo2máx en bomberos. Psicothema, 22(1), 131-136.

Sánchez, P. (julio 4 de 2017). Capacidades condicionales en el entrenamiento [información en página web]. Recuperado de https://mundoentrenamiento.com/capacidades-condicionales-en-el-entrenamiento/ 